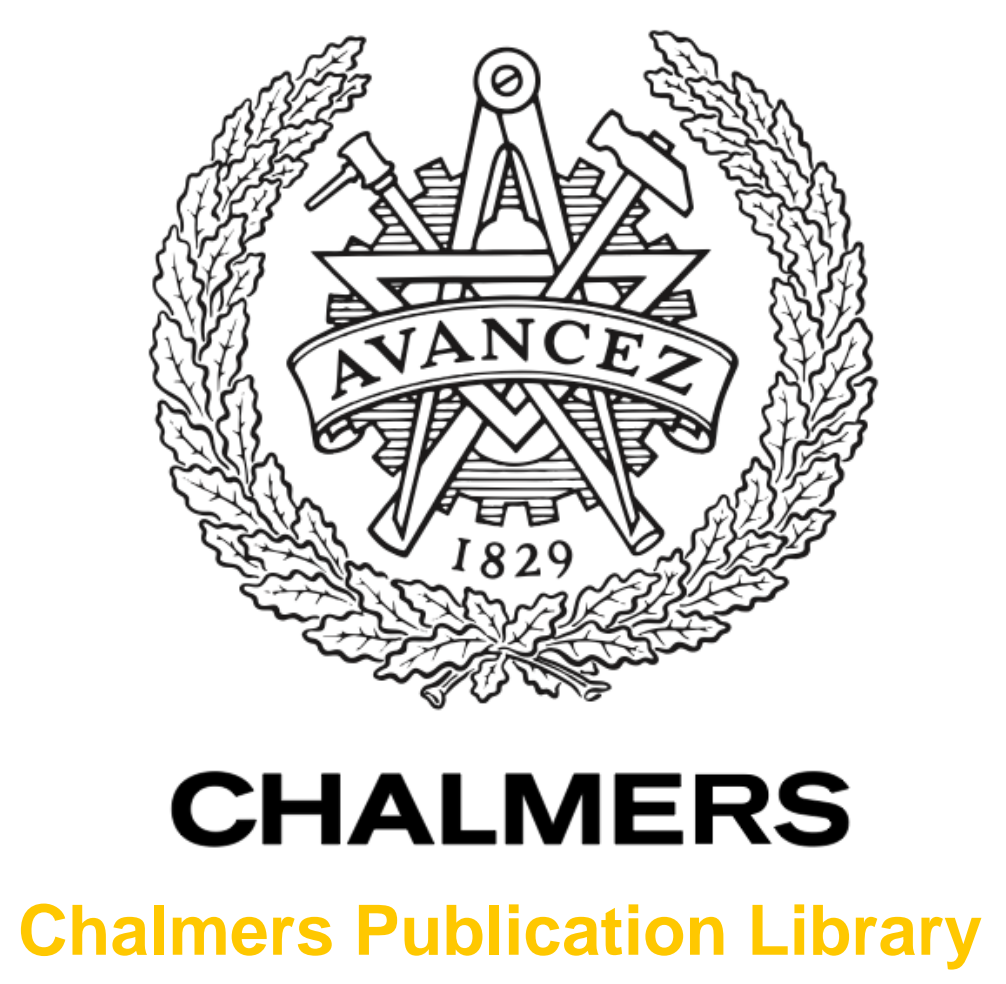

Ultrasonic wave propagation in an anisotropic cladding with a wavy interface

This document has been downloaded from Chalmers Publication Library $(\mathrm{CPL})$. It is the author's version of a work that was accepted for publication in:

Wave motion (ISSN: 0165-2125)

Citation for the published paper:

Krasnova, T. ; Jansson, P. ; Boström, A. (2005) "Ultrasonic wave propagation in an anisotropic cladding with a wavy interface". Wave motion, vol. 41(2), pp. 163-177.

http://dx.doi.org/10.1016/j.wavemoti.2004.06.005

Downloaded from: http://publications.lib.chalmers.se/publication/7170

Notice: Changes introduced as a result of publishing processes such as copy-editing and formatting may not be reflected in this document. For a definitive version of this work, please refer to the published source. Please note that access to the published version might require a subscription. 


\title{
ULTRASONIC WAVE PROPAGATION IN AN ANISOTROPIC CLADDING WITH A WAVY INTERFACE
}

\author{
TATIANA KRASNOVA, PER-ÅKE JANSSON, AND ANDERS BOSTRÖM
}

\begin{abstract}
The propagation of ultrasonic waves in a thick plate with a cladding is investigated in the two-dimensional case. The surfaces of the plate are traction-free except where an ultrasonic probe is attached and emits waves into the plate. The plate is made of two different materials, the base material and the cladding, and these are both allowed to be anisotropic. The interface between the base material and the cladding is assumed to be wavy (sinusoidal) and this is common in practice for welded claddings. The null field approach is used to solve the wave propagation problem. Thus the starting point is the (surface) integral representations in the two materials. The Green's tensors are chosen as the half space Green's tensors as only the integrals along the interface then remain. The Green's functions are expanded in Fourier transforms along the interface and the surface fields are likewise expanded. Applying the periodicity of the interface a discretized set of equations remains. For the sinusoidal interface all integrals can be computed analytically which leads to an efficient numerical scheme. Some numerical results show the influence of the anisotropy and the wavy interface.
\end{abstract}

\section{InTRODUCTION}

Ultrasonic methods are widely used in nondestructive testing, e.g. in the aerospace and nuclear power industries. A mathematical modelling of the testing situation is very valuable for a number of reasons. The modelling helps the physical intuition and in the interpretation of tests. It is also very easy to perform parametric studies with a model and it is therefore a valuable tool in the development and qualification of testing procedures.

The generation, propagation, and scattering of ultrasonic waves in elastic solids (often called elastic waves) have attracted a lot of interest during the years, both from the theoretical and experimental sides. Modelling has been performed for many situations and there now exists a number of models that include the whole chain from the generation, propagation, scattering, to the detection. These models all have their limitations, like twodimensionality, approximate ray-tracing, or Kirchhoff scattering. Most models only deal with isotropic media, although there are of course important situations where anisotropy is an essential feature.

One situation of particular importance in the nuclear power industry is that of a plate or pipe that has a cladding, usually for corrosion protection. These claddings can be fabricated in different ways. One common way is to apply an austenitic cladding on a ferritic base material by a welding process. From an ultrasonic testing point of view this leads to at least two difficulties. The austenitic cladding is anisotropic with the complications this lead to. The material can also be inhomogeneous to some degree, 
particularly through varying orientation of the crystal axes. More importantly, the interface between the cladding and the base is usually corrugated, and this can lead to strong effects on the ultrasonic properties. Hudgell [1] gives a description of austenitic clad components and of the ultrasonic testing of such components.

The intention here is to model the propagation of ultrasound in a clad component taking both the anisotropy and the interface corrugation into account. Both because this is realistic and because it simplifies the analysis the interface is taken to be sinusoidal and thus periodic. It is noted that periodic interfaces are treated extensively in the literature, particularly in electromagnetism but also for elastic waves, see, e.g. Fokkema and van den Berg [2], Fokkema [3], Glass et al. [4], Boström [5], Lakhtakia et al. [6], Roberts et al. [7], Varadan et al. [8], and El-Bahrawy [9]. However, it seems that the combination of a periodic interface and an anisotropic medium has not been investigated.

To solve the wave propagation problem in the presence of a periodic interface the null field approach is used. The starting point is then an (surface) integral representation with the Green's tensor (here half-space Green's tensors are actually used). The Green's tensor in the anisotropic case is only known in the form of integral repesentations (Fourier integrals or similar), but this is exactly what is needed in the null field approach. In contrast, in traditional integral equation approaches (Fokkema and van den Berg [2], Glass et al. [4], Roberts et al. [7]) it is much more efficient (or even essential) to use the closed form of the Green's tensor. The null field approach is used by Boström [5], Lakhtakia et al. [6], and Varadan et al. [8] to treat elastic wave propagation and scattering problems for periodic surfaces.

\section{Problem Formulation}

Consider the 2D wave propagation problem as depicted in Fig. 1. A plate of total thickness $d_{1}+d_{2}$ is made of two different anisotropic materials. The interface between the materials is assumed to be periodically corrugated with a period $a$. Later the interface will be taken as sinusoidal, but this assumption is not essential for most of what follows. An $x_{1} x_{2}$ coordinate system is introduced with the origin in the middle of the interface, the $x_{1}$ axis along the plate surfaces and the $x_{2}$ axis perpendicular to them. The interface is denoted by $S_{0}$ and its unit normal $\hat{n}_{0}$ is pointing into the upper material. The upper and lower plate surfaces are denoted by $S_{1}$ and $S_{2}$, respectively, and their unit normals are $\hat{n}_{1}$ and $\hat{n}_{2}$, respectively (both parallel to the unit vector in the $x_{2}$ direction, of course).

The material parameters and other quantities in the two materials are denoted by an upper index 1 or 2 . Thus the densities are $\rho^{1}$ and $\rho^{2}$ and the stiffnesses are $c_{j m j^{\prime} m^{\prime}}^{1}$ and $c_{j m j^{\prime} m^{\prime}}^{2}$. Here the tensor indices run over $j=1,2$, etc, and the summation convention is adopted for the tensor indices (but not for the mode indices that will appear shortly). It is assumed that the $x_{1} x_{2}$ plane is a plane of elastic symmetry of the material, so that an inplane 2D wave propagation problem is possible. However, it is not assumed that the $x_{1}$ and $x_{2}$ axes are crystal axes of the material. Typically, the materials can be orthotropic with tilted crystal axes in the $x_{1} x_{2}$ plane. 


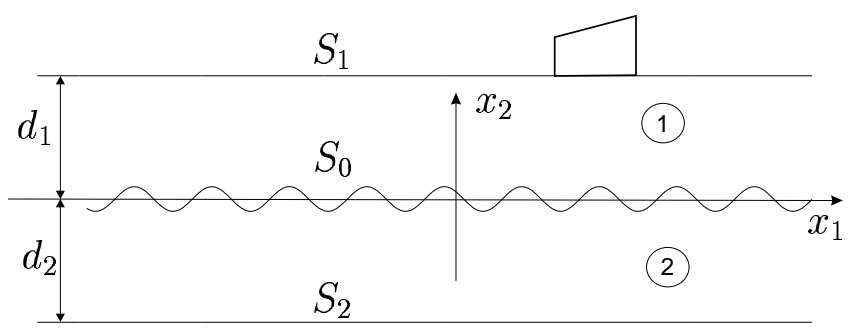

FIgURE 1. The 2D geometry with a thick plate consisting of two anisotropic materials with a wavy interface and an ultrasonic probe.

Only time harmonic conditions are considered. The time factor $\exp (-i \omega t)$, where $\omega$ is the angular frequency and $t$ is time, is suppressed throughout. The elastodynamic equation of motion can then be written

$$
\frac{\partial}{\partial x_{m}} \sigma_{m j}^{i}+\rho^{i} \omega^{2} u_{j}^{i}=0
$$

in the two materials $i=1,2$. Here $u_{j}^{i}$ is the displacement and $\sigma_{m j}^{i}$ is the stress tensor, which is related to the displacement by the constitutive equation

$$
\sigma_{m j}^{i}=c_{m j m^{\prime} j^{\prime}}^{i} \frac{\partial}{\partial x_{j^{\prime}}} u_{m^{\prime}}^{i}
$$

The surfaces $S_{1}$ and $S_{2}$ of the plate are assumed to be traction-free except that ultrasound is excited in the plate by an ultrasonic contact probe situated on the upper surface $S_{1}$. The probe is modelled by the traction it exerts on the plate, more is said about this later on. The two materials of the plate are in perfect contact so both displacement and traction are continuous along the interface $S_{0}$. Finally, to fully specify the wave propagation problem all waves must be outgoing at infinity (this means that the group velocity must be outgoing).

When employing the null field approach the starting point is an integral representation instead of the differential equation. To this end the Green's tensor $G_{j j^{\prime}}^{i}\left(\mathbf{x} ; \mathbf{x}^{\prime}\right)$ of material $i$ is introduced as the solution to the differential equation

$$
\frac{\partial}{\partial x_{m}} \Sigma_{m j l}^{i}\left(\mathbf{x} ; \mathbf{x}^{\prime}\right)+\rho^{i} \omega^{2} G_{j l}^{i}\left(\mathbf{x} ; \mathbf{x}^{\prime}\right)=-\delta_{j l} \delta\left(\mathbf{x}-\mathbf{x}^{\prime}\right),
$$

where $\delta_{j l}$ is the Kronecker delta and $\delta\left(\mathbf{x}-\mathbf{x}^{\prime}\right)$ is th $2 \mathrm{D}$ delta 'function'. The Green's stress triadic has the same relation to the Green's tensor as the stress tensor has to the displacement

$$
\Sigma_{m j l}^{i}\left(\mathbf{x} ; \mathbf{x}^{\prime}\right)=c_{m j m^{\prime} j^{\prime}}^{i} \frac{\partial}{\partial x_{j^{\prime}}} G_{m^{\prime} l}^{i}\left(\mathbf{x} ; \mathbf{x}^{\prime}\right)
$$

Note that the derivatives in Eqs. (3) and (4) are with the respect to the first argument in the Green's tensor.

The (surface) integral representations are obtained in the usual way by multiplying Eq. (1) by $G_{j l}^{i}\left(\mathbf{x} ; \mathbf{x}^{\prime}\right)$ and Eq. (3) by $u_{j}^{i}(\mathbf{x})$, integrating the difference of the obtained 
equations over the volume of material $i$, and using Gauss' theorem to obtain

$$
\begin{gathered}
(-1)^{i} \int_{S_{i}-S_{0}}\left[\Sigma_{m j l}^{i}\left(\mathbf{x} ; \mathbf{x}^{\prime}\right) u_{j}(\mathbf{x})-G_{j l}^{i}\left(\mathbf{x} ; \mathbf{x}^{\prime}\right) \sigma_{m j}(\mathbf{x})\right] n_{m} d S \\
= \begin{cases}u_{l}\left(\mathbf{x}^{\prime}\right), & \text { inside material } i, \\
0, & \text { outside material } i .\end{cases}
\end{gathered}
$$

The factor $(-1)^{i}$ in front of the integral is to take care of the different directions of the surface normals in the two materials. The surface normal component $n_{m}$ is taken for the surface in question, of course.

The Green's tensor in material $i$ is now chosen as the half-space Green's tensor which satisfies traction-free boundary conditions on $S_{i}$. Using also the traction-free boundary conditions on $S_{i}$ for the fields, the integrals over $S_{1}$ and $S_{2}$ disappear in Eq. (5) except that the integral over $S_{1}$ yields the incoming field $u_{j}^{\text {in }}$, which thus appears as a term of its own. Note that the incoming field is the one that would exist in material 1 if it were a homogeneous half-space.

\section{The Green's tensor}

The elementary plane wave solutions to Eq. (1) can be obtained by solving the dispersion relation (in $2 \mathrm{D}$ the dispersion relation can be solved analytically for simple untilted materials). However, a reformulation to a generalized eigenvalue problem is performed (this being even more convenient in $3 \mathrm{D}$ ). To this end the following vector is introduced:

$$
\mathbf{v}=\left(\begin{array}{c}
u_{1} \\
u_{2} \\
\sigma_{21} \\
\sigma_{22}
\end{array}\right)
$$

where the two traction components in the $x_{2}$ direction supplement the two displacement components. Assuming a dependence $\exp \left(i\left(q x_{1}+h x_{2}\right)\right)$ for the components of $\mathbf{v}$, Eqs. (1) and (2) give

$$
A \mathbf{v}=h B \mathbf{v}
$$

where $h$ is the eigenvalue and $\mathbf{v}$ is the eigenvector in the generalized eigenvalue problem. The matrices $A$ and $B$ depend on $q$, the angular frequency $\omega$, and the material parameters:

$$
\begin{gathered}
A=\left(\begin{array}{cccc}
c_{16} q & c_{66} q & i & 0 \\
c_{12} q & c_{26} q & 0 & i \\
c_{11} q^{2}-\rho \omega^{2} & c_{16} q^{2} & 0 & 0 \\
0 & -\rho \omega^{2} & -i q & 0
\end{array}\right) \\
B=\left(\begin{array}{cccc}
-c_{66} & -c_{26} & 0 & 0 \\
-c_{26} & -c_{22} & 0 & 0 \\
-c_{16} q & -c_{12} q & i & 0 \\
0 & 0 & 0 & i
\end{array}\right)
\end{gathered}
$$


where the stiffnesses now are given in abbreviated notation. The solutions to Eq. (7) are denoted $h_{n}^{+}$and $h_{n}^{-}$, where $n=1,2$ is a mode index and the superscript indicates if the corresponding plane wave is up- or down-going (as determined by the group velocity). The corresponding eigenvector is denoted

$$
\mathbf{v}_{n}=\left(\begin{array}{c}
U_{n 1} \\
U_{n 2} \\
T_{n 1} \\
T_{n 2}
\end{array}\right)
$$

with a superscript + or - as appropriate. To discriminate between the solutions in the two media a superscript $i(=1,2)$ is added: $h_{n}^{i \pm}, U_{n j}^{i \pm}, T_{n j}^{i \pm}$.

Using the symmetries the free space Green's tensor can be expressed as a Fourier transform in $x_{1}$ :

$$
G_{j l}^{\mathrm{free}, i}\left(x_{1}, x_{2} ; x_{1}^{\prime}, x_{2}^{\prime}\right)=\int_{-\infty}^{\infty} \sum_{n=1}^{2} D_{n}^{i \pm} U_{n l}^{i \pm} U_{n j}^{i \pm} \mathrm{e}^{i\left(q\left(x_{1}-x_{1}^{\prime}\right)+h_{n}^{i \pm}\left(x_{2}-x_{2}^{\prime}\right)\right)} d q
$$

with the \pm sign for $x_{2} \geq x_{2}^{\prime}$. The coefficients $D_{n}^{i \pm}$ are integration constants that are determined by the jump conditions that the Green's tensor must satisfy at $x_{2}=x_{2}^{\prime}$, which give

$$
\begin{gathered}
\sum_{n=1}^{2}\left(D_{n}^{i+} U_{n j}^{i+} U_{n l}^{i+}-D_{n}^{i-} U_{n j}^{i-} U_{n l}^{i-}\right)=0, \quad j, l=1,2, \\
\sum_{n=1}^{2}\left(D_{n}^{i+} T_{n j}^{i+} U_{n l}^{i+}-D_{n}^{i-} T_{n j}^{i-} U_{n l}^{i-}\right)=-\frac{\delta_{j l}}{2 \pi}, \quad j, l=1,2 .
\end{gathered}
$$

There are actually eight equations for the four unknowns $D_{n}^{i+}$ and $D_{n}^{i-}, n=1,2$, for each $i$. This situation arises because the symmetries of the Green's tensor have been exploited when writing Eq. (11). A good choice seems to be to use only the equations with $j=l$ in Eqs. (12) and (13).

To obtain the half-space $x_{2}<d_{1}$ Green's tensor for material $i=1$, a reflected part is added to the free space Green's tensor

$$
\begin{aligned}
& G_{j l}^{1}\left(x_{1}, x_{2} ; x_{1}^{\prime}, x_{2}^{\prime}\right)=\int_{-\infty}^{\infty} \sum_{n=1}^{2} D_{n}^{1 \pm} U_{n l}^{1 \pm} U_{n j}^{1 \pm} \mathrm{e}^{i\left(q\left(x_{1}-x_{1}^{\prime}\right)+h_{n}^{1 \pm}\left(x_{2}-x_{2}^{\prime}\right)\right)} d q \\
& \quad+\int_{-\infty}^{\infty} \sum_{n, n^{\prime}=1}^{2} D_{n}^{1+} U_{n l}^{1+} R_{n n^{\prime}}^{1} U_{n^{\prime} j}^{1-} \mathrm{e}^{i\left(q\left(x_{1}-x_{1}^{\prime}\right)+h_{n^{\prime}}^{1-}\left(x_{2}-d_{1}\right)-h_{n}^{1+}\left(x_{2}^{\prime}-d_{1}\right)\right)} d q
\end{aligned}
$$


for $x_{2} \geq x_{2}^{\prime}$. The reflection matrix is obtained from the stress-free boundary condition at $x_{2}=d_{1}$ :

$$
T_{n j}^{1+}+\sum_{n^{\prime}=1}^{2} R_{n n^{\prime}}^{1} T_{n^{\prime} j}^{1-}=0, \quad n, j=1,2 .
$$

The reflection matrix is essentially composed of the ordinary reflection coefficients, although it of course depends on the normalization of the plane waves.

For material $i=2$ the half-space Green's tensor for $x_{2}>-d_{2}$ is similarly

$$
\begin{aligned}
& G_{j l}^{2}\left(x_{1}, x_{2} ; x_{1}^{\prime}, x_{2}^{\prime}\right)=\int_{-\infty}^{\infty} \sum_{n=1}^{2} D_{n}^{2 \pm} U_{n l}^{2 \pm} U_{n j}^{2 \pm} \mathrm{e}^{i\left(q\left(x_{1}-x_{1}^{\prime}\right)+h_{n}^{2 \pm}\left(x_{2}-x_{2}^{\prime}\right)\right)} d q \\
& \quad+\int_{-\infty}^{\infty} \sum_{n, n^{\prime}=1}^{2} D_{n}^{2-} U_{n l}^{2-} R_{n n^{\prime}}^{2} U_{n^{\prime} j}^{2+} \mathrm{e}^{i\left(q\left(x_{1}-x_{1}^{\prime}\right)+h_{n^{\prime}}^{2+}\left(x_{2}+d_{2}\right)-h_{n}^{2-}\left(x_{2}^{\prime}+d_{2}\right)\right)} d q .
\end{aligned}
$$

The reflection matrix is determined from

$$
T_{n j}^{2-}+\sum_{n^{\prime}=1}^{2} R_{n n^{\prime}}^{2} T_{n^{\prime} j}^{2+}=0, \quad n, j=1,2 .
$$

The corresponding Green's stress tensors are obtained according to Eq. (4). For example, for the first term in Eq. (16) this amounts to exchanging $U_{n j}^{2 \pm}$ for

$$
S_{n m j}^{2 \pm}=i\left(q c_{m j j^{\prime} 1}^{2}+h_{n}^{2 \pm} c_{m j j^{\prime} 2}^{2}\right) U_{n j^{\prime}}^{2 \pm} .
$$

The other terms in the Green's tensors are treated in the same way.

\section{The NUll FIELD APPROACH}

To proceed the expansion of the Green's tensor in Eq. (14) is now inserted into the integral representation (5). Then the two cases when the field point is inside or outside material 1 must be distinguished. It is also noted that the Green's function expansion is only valid in the half-space $x_{2}<d_{1}$. Letting the field point $\mathbf{x}^{\prime}$ lie in material 1 in the region above the maximum of the interface $S_{0}$ (so that $x_{2}^{\prime}>x_{2}$ in the free space part of the Green's tensor expansion (14)), the following representation is obtained for the scattered field $u_{j}^{\text {sc1 }}=u_{j}^{1}-u_{j}^{\text {in }}$ (use also the property $G_{j l}\left(x_{1}, x_{2} ; x_{1}^{\prime}, x_{2}^{\prime}\right)=G_{l j}\left(x_{1}^{\prime}, x_{2}^{\prime} ; x_{1}, x_{2}\right)$ ):

$$
u_{l}^{\mathrm{sc} 1}\left(x_{1}, x_{2}\right)=\int_{-\infty}^{\infty} \sum_{n=1}^{2} f_{n}^{1}(q)\left[U_{n l}^{1+} \mathrm{e}^{i h_{n}^{1+} x_{2}}+\sum_{n^{\prime}=1}^{2} R_{n n^{\prime}}^{1} U_{n^{\prime} l}^{1-} \mathrm{e}^{i\left(h_{n^{\prime}}^{1-}\left(x_{2}-d_{1}\right)+h_{n}^{1+} d_{1}\right)}\right] \mathrm{e}^{i q x_{1}} d q
$$

where

$$
f_{n}^{1}(q)=\int_{S_{0}}\left[n_{m}\left(-S_{n m j}^{1+}\right) u_{j}\left(x_{1}, x_{2}\right)-U_{n j}^{1+} t_{j}\left(x_{1}, x_{2}\right)\right] D_{n}^{1+} \mathrm{e}^{-i\left(q x_{1}+h_{n}^{1+} x_{2}\right)} d S .
$$

Here $u_{j}$ and $t_{j}=n_{m} \sigma_{m j}$ are the (unknown) displacement and traction on $S_{0}$. Due to the interface conditions the displacement and traction are of course the same on both sides of $S_{0}$. 
Letting the field point lie below the minimum of the interface $S_{0}$ in the integral representation (5) gives an expansion of the incoming field

$$
u_{l}^{\mathrm{in}}\left(x_{1}, x_{2}\right)=\int_{-\infty}^{\infty} \sum_{n=1}^{2} \xi_{n}(q) U_{n l}^{1-} \mathrm{e}^{i\left(q x_{1}+h_{n}^{1-}\left(x_{2}-d_{1}\right)\right)} d q
$$

where

$$
\begin{aligned}
\xi_{n}(q) & =-\int_{S_{0}}\left(\left[D_{n}^{1-}\left(-S_{n m j}^{1-}\right) \mathrm{e}^{i h_{n}^{1-}\left(d_{1}-x_{2}\right)}+\sum_{n^{\prime}=1}^{2} D_{n^{\prime}}^{1+}\left(-S_{n^{\prime} m j}^{1+}\right) R_{n^{\prime} n}^{1} \mathrm{e}^{i h_{n^{\prime}}^{1+}\left(d_{1}-x_{2}\right)}\right] n_{m} u_{j}\left(x_{1}, x_{2}\right)\right. \\
& \left.-\left[D_{n}^{1-} U_{n j}^{1-} \mathrm{e}^{i h_{n}^{1-}\left(d_{1}-x_{2}\right)}+\sum_{n^{\prime}=1}^{2} D_{n^{\prime}}^{1+} U_{n^{\prime} j}^{1+} R_{n^{\prime} n}^{1} \mathrm{e}^{i h_{n^{\prime}}^{1+}\left(d_{1}-x_{2}\right)}\right] t_{j}\left(x_{1}, x_{2}\right)\right) \mathrm{e}^{-i q x_{1}} d S .
\end{aligned}
$$

Here the unknown displacement and traction on $S_{0}$ again enter. As the incoming field is given by specifying the probe, the expansion coefficients $\xi_{n}$ can be regarded as known quantities.

Turning to material 2, the same procedure is repeated. Inserting the Green's tensor expansion (16) into the integral representation (5) with the field point in material 2 below the minimum of the interface $S_{0}$ gives

$$
u_{l}^{2}\left(x_{1}, x_{2}\right)=\int_{-\infty}^{\infty} \sum_{n=1}^{2} f_{n}^{2}(q)\left[U_{n l}^{2-} \mathrm{e}^{i h_{n}^{2-} x_{2}}+\sum_{n^{\prime}=1}^{2} R_{n n^{\prime}}^{2} U_{n^{\prime} l}^{2+} \mathrm{e}^{i\left(h_{n^{\prime}}^{2+}\left(x_{2}+d_{2}\right)-h_{n}^{2-} d_{2}\right)}\right] \mathrm{e}^{i q x_{1}} d q
$$

where

$$
f_{n}^{2}(q)=-\int_{S_{0}}\left[n_{m}\left(-S_{n m j}^{2-}\right) u_{j}\left(x_{1}, x_{2}\right)-U_{n j}^{2-} t_{j}\left(x_{1}, x_{2}\right)\right] D_{n}^{2-} \mathrm{e}^{-i\left(q x_{1}+h_{n}^{2-} x_{2}\right)} d S .
$$

In contrast to the situation in material 1 this is the total field (because there is no incoming field in material 2). Finally, letting the field point lie above the maximum of the interface $S_{0}$ gives

$$
\begin{aligned}
0= & -\int_{S_{0}}\left(\left[D_{n}^{2+}\left(-S_{n m j}^{2+}\right) \mathrm{e}^{-i h_{n}^{2+} x_{2}}+\sum_{n^{\prime}=1}^{2} D_{n^{\prime}}^{2-}\left(-S_{n^{\prime} m j}^{2-}\right) R_{n^{\prime} n}^{2} \mathrm{e}^{i\left(-h_{n^{\prime}}^{2-}\left(d_{2}+x_{2}\right)+h_{n}^{2+} d_{2}\right)}\right] n_{m} u_{j}\left(x_{1}, x_{2}\right)\right. \\
& \left.-\left[D_{n}^{2+} U_{n j}^{2+} \mathrm{e}^{-i h_{n}^{2+} x_{2}}+\sum_{n^{\prime}=1}^{2} D_{n^{\prime}}^{2-} U_{n^{\prime} j}^{2-} R_{n^{\prime} n}^{2} \mathrm{e}^{i\left(-h_{n^{\prime}}^{2-}\left(d_{2}+x_{2}\right)+h_{n}^{2+} d_{2}\right)}\right] t_{j}\left(x_{1}, x_{2}\right)\right) \mathrm{e}^{-i q x_{1}} d S .
\end{aligned}
$$

Equations (20), (22), (24), and (25) contain the fields $u_{j}$ and $t_{j}$ on the interface, but also the expansion coefficients $f_{n}^{1}$ and $f_{n}^{2}$ are unknowns at this stage. The equations can be solved by suitable expansions of $u_{j}$ and $t_{j}$. To obtain integrations that can be performed analytically for a sinusoidal interface it is convenient to use $x_{1}$ as the integration variable in the integrations over the interface. Let the interface be given by $x_{2}=s\left(x_{1}\right)$ where $s\left(x_{1}\right)$ 
is a differentiable function. The unit normal to the interface has the components

$$
\begin{aligned}
& n_{1}=-s^{\prime}\left(1+\left(s^{\prime}\right)^{2}\right)^{-1 / 2}, \\
& n_{2}=\left(1+\left(s^{\prime}\right)^{2}\right)^{-1 / 2},
\end{aligned}
$$

where $s^{\prime}=\frac{d}{d x_{1}} s\left(x_{1}\right)$. The integration measure is $d S=d x\left(1+\left(s^{\prime}\right)^{2}\right)^{1 / 2}$ and it is convenient to incorporate this in the expansions of the surface fields as follows:

$$
\begin{aligned}
u_{j}\left(x_{1}\right) & =\int_{-\infty}^{\infty} \alpha_{j}\left(q^{\prime}\right) \mathrm{e}^{i q^{\prime} x_{1}} d q^{\prime}, \\
\left(1+\left(s^{\prime}\right)^{2}\right)^{1 / 2} t_{j}\left(x_{1}\right) & =\int_{-\infty}^{\infty} \beta_{j}\left(q^{\prime}\right) \mathrm{e}^{i q^{\prime} x_{1}} d q^{\prime} .
\end{aligned}
$$

As the integrals containing $u_{j}$ also contain $n_{m}$ but those containing $t_{j}$ do not contain $n_{m}$, this means that the factor $\left(1+\left(s^{\prime}\right)^{2}\right)^{1 / 2}$ does not appear in any of the integrals. It is now possible to insert Eqs. (28) and (29) into Eqs. (22), (25), (20), and (24). In general this leads to double integrals without any possibilities of simplification (the expressions are not given as they are not very enlightening). However, it is noted that the developments so far have not made any use of the periodicity of the interface.

\section{The PERIODIC INTERFACE}

To proceed the periodicity of the interface is now exploited. If the function $g(x)$ is periodic with period $a$ the following relation is valid

$$
\int_{-\infty}^{\infty} g(x) \mathrm{e}^{i q x} d x=\sum_{p^{\prime \prime}=-\infty}^{\infty} \delta\left(\frac{q a}{2 \pi}+p^{\prime \prime}\right) \int_{0}^{a} g(x) \mathrm{e}^{i q x} d x
$$

When Eqs. (28) and (29) are inserted into Eq. (22), and Eq. (30) is used the result is

$$
\begin{aligned}
\xi_{n}(q) & =\sum_{p^{\prime \prime}=-\infty}^{\infty} \int_{0}^{a}\left(\left[D_{n}^{1-} S_{n m j}^{1-} \mathrm{e}^{i h_{n}^{1-}\left(d_{1}-s\left(x_{1}\right)\right)}\right.\right. \\
& \left.+\sum_{n^{\prime}=1}^{2} D_{n^{\prime}}^{1+} S_{n^{\prime} m j}^{1+} R_{n^{\prime} n}^{1} \mathrm{e}^{i h_{n^{\prime}}^{1+}\left(d_{1}-s\left(x_{1}\right)\right)}\right] \bar{n}_{m} \alpha_{j}\left(q-\frac{2 \pi p^{\prime \prime}}{a}\right)+\left[D_{n}^{1-} U_{n j}^{1-} \mathrm{e}^{i h_{n}^{1-}\left(d_{1}-s\left(x_{1}\right)\right)}\right. \\
& \left.\left.+\sum_{n^{\prime}=1}^{2} D_{n^{\prime}}^{1+} U_{n^{\prime} j}^{1+} R_{n^{\prime} n}^{1} \mathrm{e}^{i h_{n^{\prime}}^{1+}\left(d_{1}-s\left(x_{1}\right)\right)}\right] \beta_{j}\left(q-\frac{2 \pi p^{\prime \prime}}{a}\right)\right) \mathrm{e}^{-2 \pi i p^{\prime \prime} x_{1} / a} d x_{1} .
\end{aligned}
$$

Here $\bar{n}_{1}=-s^{\prime}\left(x_{1}\right)$ and $\bar{n}_{2}=1$ are unnormalized components of the normal to the interface $S_{0}$. It must be remembered that $h_{n}^{1-}, D_{n}^{1-}, S_{n m j}^{1-}, R_{n n^{\prime}}^{1}$, etc, all are functions of $q$ although this dependence is not explicitly shown. It is seen in Eq. (31) that $\xi_{n}(q), \alpha_{j}\left(q-2 \pi p^{\prime \prime} / a\right)$ and $\beta_{j}\left(q-2 \pi p^{\prime \prime} / a\right)$ only couple for $q$ values that differ by a multiple of $2 \pi / a$. It is therefore 
natural to write $q=q_{0}+2 \pi p / a$, with $\left|q_{0}\right| \leq \pi / a$, and to introduce

$$
\begin{gathered}
\xi_{n p}\left(q_{0}\right)=\xi_{n}\left(q_{0}+\frac{2 \pi p}{a}\right), \\
\alpha_{n p^{\prime}}\left(q_{0}\right)=\alpha_{n}\left(q_{0}+\frac{2 \pi p^{\prime}}{a}\right), \\
\beta_{n p^{\prime}}\left(q_{0}\right)=\beta_{n}\left(q_{0}+\frac{2 \pi p^{\prime}}{a}\right),
\end{gathered}
$$

where $p^{\prime}=p-p^{\prime \prime}$. Summing over $p^{\prime}$ instead of $p^{\prime \prime}$, Eq. (31) then becomes

$$
\xi_{n p}=\sum_{p^{\prime}=-\infty}^{\infty}\left(Q_{n p j p^{\prime}}^{1} \alpha_{j p^{\prime}}+Q_{n p j p^{\prime}}^{2} \beta_{j p^{\prime}}\right) .
$$

Here it must be remembered that there is also a summation over the tensor index $j$. Here and in the following the dependence on $q_{0}$ is suppressed. The matrices are

$$
\begin{aligned}
Q_{n p j p^{\prime}}^{1} & =\int_{0}^{a}\left(D_{n p}^{1-} S_{n m j p}^{1-} \mathrm{e}^{i h_{n p}^{1-}\left(d_{1}-s\left(x_{1}\right)\right)}\right. \\
& \left.+\sum_{n^{\prime}=1}^{2} D_{n^{\prime} p}^{1+} S_{n^{\prime} m j p}^{1+} R_{n^{\prime} n p}^{1} \mathrm{e}^{i h_{n^{\prime} p}^{1+}\left(d_{1}-s\left(x_{1}\right)\right)}\right) \bar{n}_{m} \mathrm{e}^{2 \pi i\left(p^{\prime}-p\right) x_{1} / a} d x_{1} \\
Q_{n p j p^{\prime}}^{2} & =\int_{0}^{a}\left(D_{n p}^{1-} U_{n j p}^{1-} \mathrm{e}^{i h_{n p}^{1-}\left(d_{1}-s\left(x_{1}\right)\right)}\right. \\
& \left.+\sum_{n^{\prime}=1}^{2} D_{n^{\prime} p}^{1+} U_{n^{\prime} j p}^{1+} R_{n^{\prime} n p}^{1} \mathrm{e}^{i h_{n^{\prime} p}^{1+}\left(d_{1}-s\left(x_{1}\right)\right)}\right) \mathrm{e}^{2 \pi i\left(p^{\prime}-p\right) x_{1} / a} d x_{1} .
\end{aligned}
$$

Here (cf. Eqs. (32)-(34))

$$
h_{n p}^{1-}=h_{n p}^{1-}\left(q_{0}\right)=h_{n}^{1-}\left(q_{0}+\frac{2 \pi p}{a}\right)
$$

and similarly for the other quantities having an index $p$.

In the same way Eqs. (25), (20), and (24) give

$$
\begin{aligned}
0 & =\sum_{p^{\prime}=-\infty}^{\infty}\left(Q_{n p j p^{\prime}}^{3} \alpha_{j p^{\prime}}+Q_{n p j p^{\prime}}^{4} \beta_{j p^{\prime}}\right), \\
f_{n p}^{1} & =\sum_{p^{\prime}=-\infty}^{\infty}\left(P_{n p j p^{\prime}}^{1} \alpha_{j p^{\prime}}+P_{n p j p^{\prime}}^{2} \beta_{j p^{\prime}}\right), \\
f_{n p}^{2} & =\sum_{p^{\prime}=-\infty}^{\infty}\left(P_{n p j p^{\prime}}^{3} \alpha_{j p^{\prime}}+P_{n p j p^{\prime}}^{4} \beta_{j p^{\prime}}\right) .
\end{aligned}
$$


The matrices are

$$
\begin{aligned}
Q_{n p j p^{\prime}}^{3} & =\int_{0}^{a}\left(D_{n p}^{2+} S_{n m j p}^{2+} \mathrm{e}^{-i h_{n p}^{2+} s\left(x_{1}\right)}\right. \\
& \left.+\sum_{n^{\prime}=1}^{2} D_{n^{\prime} p}^{2-} S_{n^{\prime} m j p}^{2-} R_{n^{\prime} n p}^{2} \mathrm{e}^{i\left(-h_{n^{\prime} p}^{2-}\left(d_{2}+s\left(x_{1}\right)\right)+h_{n p}^{2+} d_{2}\right)}\right) \bar{n}_{m} \mathrm{e}^{2 \pi i\left(p^{\prime}-p\right) x_{1} / a} d x_{1}, \\
Q_{n p j p^{\prime}}^{4} & =\int_{0}^{a}\left(D_{n p}^{2+} U_{n j p}^{2+} \mathrm{e}^{-i h_{n p}^{2+} s\left(x_{1}\right)}\right. \\
& \left.+\sum_{n^{\prime}=1}^{2} D_{n^{\prime} p}^{2-} U_{n^{\prime} j p}^{2-} R_{n^{\prime} n p}^{2} \mathrm{e}^{i\left(-h_{n^{\prime} p}^{2-}\left(d_{2}+s\left(x_{1}\right)\right)+h_{n p}^{2+} d_{2}\right)}\right) \mathrm{e}^{2 \pi i\left(p^{\prime}-p\right) x_{1} / a} d x_{1}, \\
P_{n p j p^{\prime}}^{1} & =-\int_{0}^{a} D_{n p}^{1+} S_{n m j p}^{1+} \bar{n}_{m} \mathrm{e}^{i\left(-h_{n p}^{1+} s\left(x_{1}\right)+2 \pi\left(p^{\prime}-p\right) x_{1} / a\right)} d x_{1}, \\
P_{n p j p^{\prime}}^{2}= & -\int_{0}^{a} D_{n p}^{1+} U_{n j p}^{1+} \mathrm{e}^{i\left(-h_{n p}^{1+} s\left(x_{1}\right)+2 \pi\left(p^{\prime}-p\right) x_{1} / a\right)} d x_{1}, \\
P_{n p j p^{\prime}}^{3}= & \int_{0}^{a} D_{n p}^{2-} S_{n m j p}^{2-} \bar{n}_{m} \mathrm{e}^{i\left(-h_{n p}^{2-} s\left(x_{1}\right)+2 \pi\left(p^{\prime}-p\right) x_{1} / a\right)} d x_{1}, \\
P_{n p j p^{\prime}}^{4}= & \int_{0}^{a} D_{n p}^{2-} U_{n j p}^{2-} \mathrm{e}^{i\left(-h_{n p}^{2-} s\left(x_{1}\right)+2 \pi\left(p^{\prime}-p\right) x_{1} / a\right)} d x_{1} .
\end{aligned}
$$

As the incoming field, and thereby $\xi_{n p}\left(q_{0}\right)$, can be regarded as known, Eqs. (35) and (39) can be solved for the unknown expansion coefficients $\alpha_{j p}$ and $\beta_{j p}$ of the surface fields. Eqs. (40) and (41) then give the expansion coefficients $f_{n p}^{1}$ and $f_{n p}^{2}$ of the scattered fields which are given by Eqs. (19) and (23). Expressed in $f_{n p}^{1}$ Eq.(19) becomes

$$
\begin{aligned}
u_{l}^{\mathrm{sc} 1}\left(x_{1}, x_{2}\right) & =\sum_{p=-\infty}^{\infty} \int_{-\pi / a}^{\pi / a} \sum_{n=1}^{2} f_{n p}^{1}\left[U_{n l p}^{1+} \mathrm{e}^{i h_{n p}^{1+} x_{2}}\right. \\
& \left.+\sum_{n^{\prime}=1}^{2} R_{n n^{\prime} p}^{1} U_{n^{\prime} l p}^{1-} \mathrm{e}^{i\left(h_{n^{\prime} p}^{1-}\left(x_{2}-d_{1}\right)+h_{n p}^{1+} d_{1}\right)}\right] \mathrm{e}^{i x_{1}\left(q_{0}+2 \pi p / a\right)} d q_{0},
\end{aligned}
$$

and similarly for Eqs. (23) and (21).

\section{The Sinusoidal interface}

The interface is now chosen to be sinusoidal:

$$
s\left(x_{1}\right)=b \sin \left(\frac{2 \pi x_{1}}{a}\right) .
$$

The main reason for this choice is that the sinusoidal shape reasonably resembles the one that appear in real applications with a welded cladding. Besides being reasonable the sinusoidal interface has the great advantage that the integrals appearing in Eqs. (36), 
(37), (42)-(47) can be calculated analytically. The following two integrals appear for the sinusoidal interface:

$$
\begin{gathered}
\int_{0}^{a} \mathrm{e}^{-i \gamma \sin 2 \pi x_{1} / a} \mathrm{e}^{2 \pi i p x_{1} / a} d x_{1}=a J_{p}(\gamma), \\
\int_{0}^{a} \cos \frac{2 \pi x_{1}}{a} \mathrm{e}^{-i \gamma \sin 2 \pi x_{1} / a} \mathrm{e}^{2 \pi i p x_{1} / a} d x_{1}=\frac{p a}{\gamma} J_{p}(\gamma) .
\end{gathered}
$$

where $J_{p}(\gamma)$ is a Bessel function. The equations are also valid for $p<0$ with $J_{-p}(\gamma)=$ $(-1)^{p} J_{p}(\gamma)$. For the sinusoidal interface the integrals now become

$$
\begin{aligned}
& Q_{n p j p^{\prime}}^{1}=A_{n p j p^{\prime}}^{1-} \mathrm{e}^{i h_{n p}^{1-} d_{1}}+\sum_{n^{\prime}=1}^{2} A_{n^{\prime} p j p^{\prime}}^{1+} R_{n^{\prime} n p}^{1} \mathrm{e}^{i h_{n^{\prime} p}^{1+} d_{1}}, \\
& Q_{n p j p^{\prime}}^{2}=B_{n p j p^{\prime}}^{1-} \mathrm{e}^{i h_{n p}^{1-} d_{1}}+\sum_{n^{\prime}=1}^{2} B_{n^{\prime} p j p^{\prime}}^{1+} R_{n^{\prime} n p}^{1} \mathrm{e}^{i h_{n^{\prime} p}^{1+} d_{1}}, \\
& Q_{n p j p^{\prime}}^{3}=A_{n p j p^{\prime}}^{2+}+\sum_{n^{\prime}=1}^{2} A_{n^{\prime} p j p^{\prime}}^{2-} R_{n^{\prime} n p}^{2} \mathrm{e}^{i d_{2}\left(h_{n p}^{2+}-h_{n^{\prime} p}^{2-}\right)}, \\
& Q_{n p j p^{\prime}}^{4}=B_{n p j p^{\prime}}^{2+}+\sum_{n^{\prime}=1}^{2} B_{n^{\prime} p j p^{\prime}}^{2-} R_{n^{\prime} n p}^{2} \mathrm{e}^{i d_{2}\left(h_{n p}^{2+}-h_{n^{\prime} p}^{2-}\right)}, \\
& P_{n p j p^{\prime}}^{1}=-A_{n p j p^{\prime}}^{1+}, \\
& P_{n p j p^{\prime}}^{2}=-B_{n p j p^{\prime}}^{1+}, \\
& P_{n p j p^{\prime}}^{3}=A_{n j p p^{\prime}}^{2-}, \\
& P_{n p j p^{\prime}}^{4}=B_{n p j p^{\prime}}^{2-},
\end{aligned}
$$

with

$$
\begin{aligned}
& A_{n p j p^{\prime}}^{i \pm}=\left(\frac{2 \pi\left(p-p^{\prime}\right)}{h_{n p}^{i \pm}} S_{n 1 j p}^{i \pm}+a S_{n 2 j p}^{i \pm}\right) J_{p^{\prime}-p}\left(b h_{n p}^{i \pm}\right) D_{n p}^{i \pm}, \quad i=1,2, \\
& B_{n p j p^{\prime}}^{i \pm}=a U_{n j p}^{i \pm} J_{p^{\prime}-p}\left(b h_{n p}^{i \pm}\right) D_{n p}^{i \pm}, \quad i=1,2 .
\end{aligned}
$$

\section{Numerical RESULtS}

In this section a few numerical results are given that illustrate the effects of the corrugated interface and the anisotropy.

The numerical computations are mostly straightforward. The integrals when computing the fields, as in Eq. (48), contain singularities in the integrands corresponding to the plate modes (in the whole plate consisting of the two materials with corrugated interface). This can be circumvented by deforming the integration contour into the complex plane, but here a small damping is introduced instead. This is both an easier way and it also corresponds to reality as all materials have some damping. The damping is introduced by giving the 


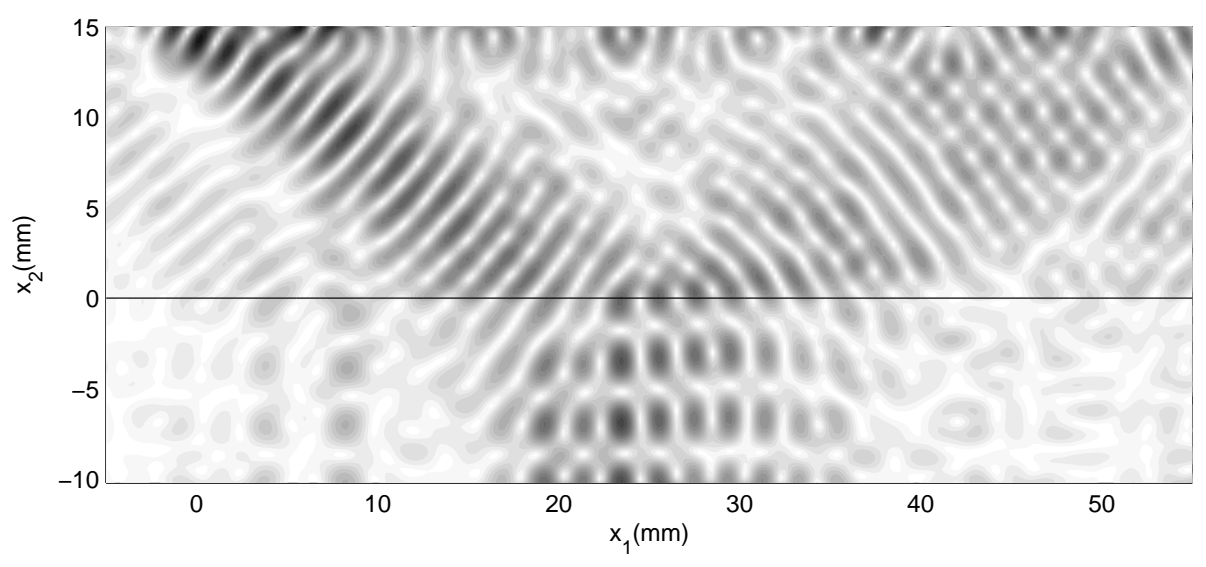

(a) $\psi=45^{\circ}$

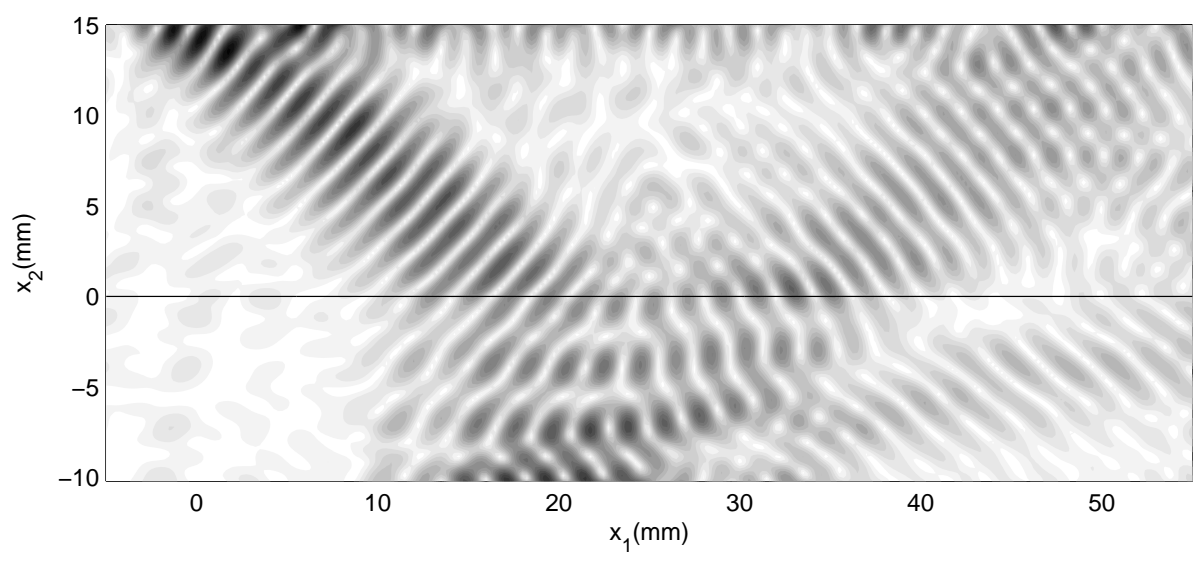

(b) $\psi=60^{\circ}$

Figure 2. The field in the plate with flat interface and two orientations of the anisotropy.

stiffness constants a small imaginary part, which has been chosen as $0.5 \%$ of the real part. All the infinite summations over $p$ and $p^{\prime}$ have to be truncated, of course. This has been checked by increasing the number of terms until stable results are established. In all the results given below it is enough to take 11 terms (i.e. $p$ goes from -5 to 5 ).

There are many parameters that can be varied in the present problem, but the focus is on illustrating the effects of the corrugation and the anisotropy and therefore only parameters related to these are varied. The upper part of the plate is chosen as an isotropic steel with density $\rho=8420 \mathrm{~kg} / \mathrm{m}^{3}$, Young's modulus $E=213 \mathrm{GPa}$, Poisson's ratio $\nu=0.29$, and thickness $d_{1}=15 \mathrm{~mm}$. The lower part is chosen as an anisotropic austenitic weld 


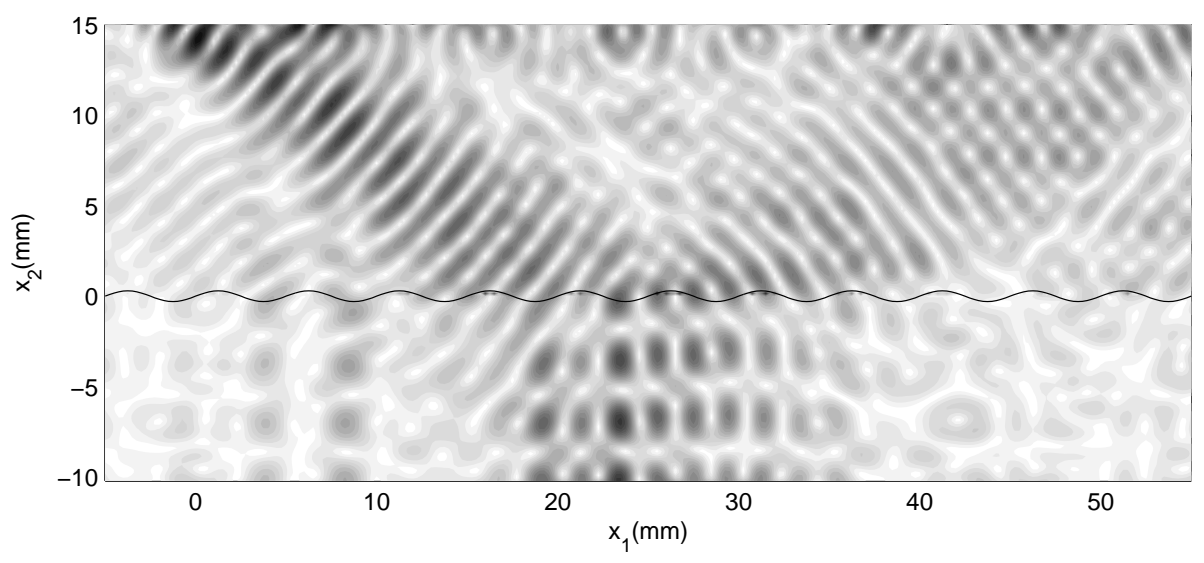

(a) $b=0.3 \mathrm{~mm}$

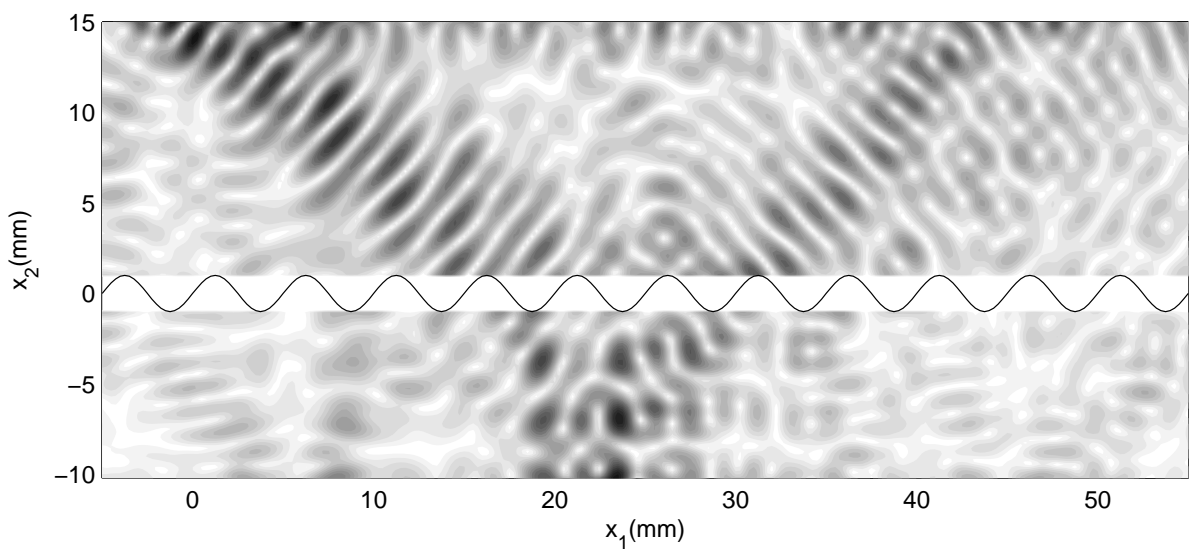

(b) $b=1 \mathrm{~mm}$

Figure 3 . The field in the plate with corrugated interface with $a=5 \mathrm{~mm}$ and two different heights $b$. Anisotropy orientation $\psi=45^{\circ}$.

material with density $\rho=8120 \mathrm{~kg} / \mathrm{m}^{3}$ and thickness $10 \mathrm{~mm}$. This material is transversely isotropic with relevant stiffness constants in abbreviated notation $C_{11}=216.0 \mathrm{GPa}, C_{22}=$ 262.7 $\mathrm{GPa}, C_{66}=129.0 \mathrm{GPa}$, and $C_{12}=145.0 \mathrm{GPa}$. To fully specify the material in this $2 \mathrm{D}$ case also the orientation of the crystal axes must be given by an angle $\psi$ measured counter-clockwise from the $x_{1}$-axis. This angle is given the two values $45^{\circ}$ and $60^{\circ}$ in the following. Also the two parameters specifying the corrugation, the period $a$ and the half height $b$, are varied.

To excite ultrasonic waves in the plate an angled SV probe is applied to the upper surface. The probe is located at $x_{1}=0$ and has width $10 \mathrm{~mm}$, angle $45^{\circ}$, and frequency $1 \mathrm{MHz}$. 


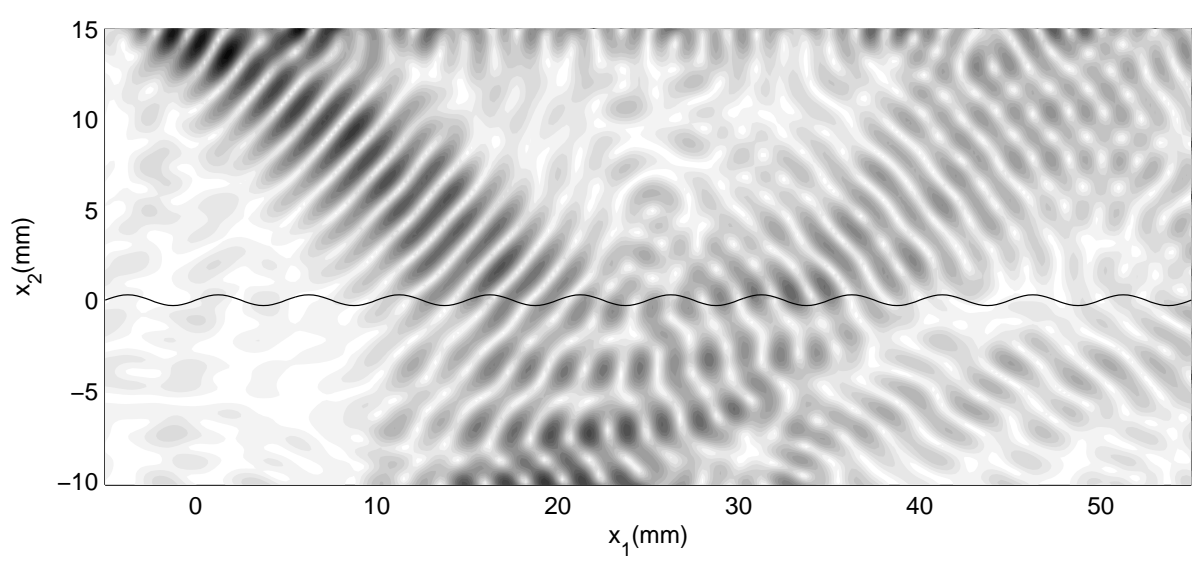

(a) $b=0.3 \mathrm{~mm}$

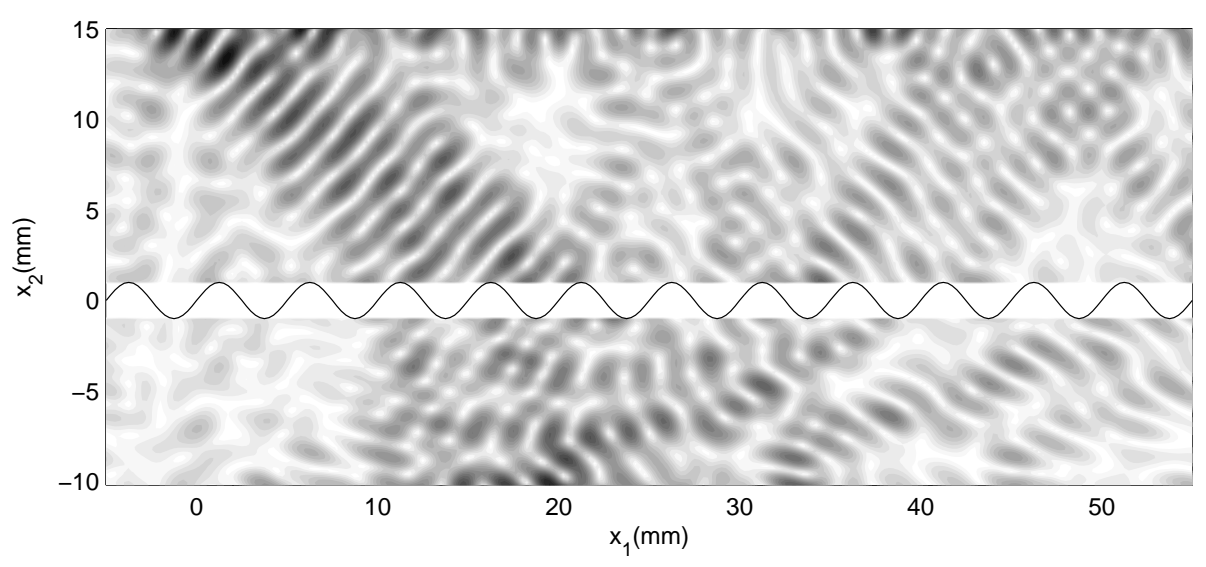

(b) $b=1 \mathrm{~mm}$

Figure 4 . The field in the plate with corrugated interface with $a=5 \mathrm{~mm}$ and two different heights $b$. Anisotropy orientation $\psi=60^{\circ}$.

The ultrasonic wavelengths in the isotropic steel are then about $3 \mathrm{~mm}$ and $6 \mathrm{~mm}$ for $\mathrm{S}$ and $\mathrm{P}$ waves, respectively, with similar but direction-dependent values in the anisotropic austenite. Results are only given at a fixed frequency, this is computationally efficient and it gives a good overall view of the field, but it does not give any travel-time information, of course. The absolute value of the real part of the displacement vector is plotted in the relevant part of the plate. A linear grey scale is used with black as the largest amplitude and white as practically zero amplitude. The scale is the same in all the plots.

One problem that arises when plotting the field in the plate is that the fields in Eqs. (19) and (23) are only valid above the top and below the bottom, respectively, of the 


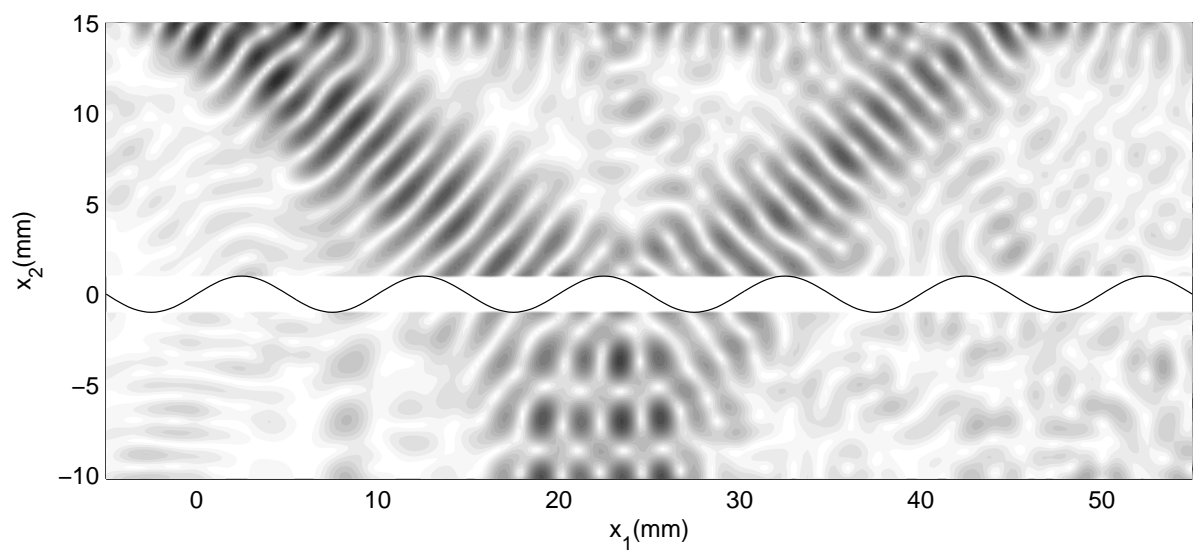

(a) $b=1 \mathrm{~mm}$

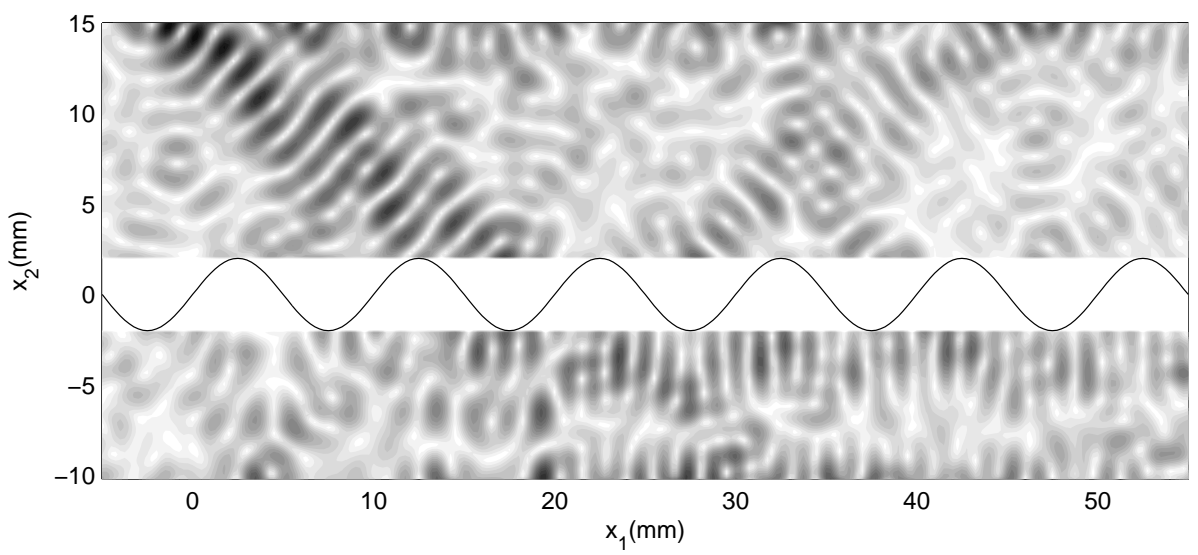

(b) $b=2 \mathrm{~mm}$

Figure 5. The field in the plate with corrugated interface with $a=10 \mathrm{~mm}$ and two different heights $b$. Anisotropy orientation $\psi=45^{\circ}$.

corrugations. For small corrugations it is possible to use the expansion all the way down to the interface as will be seen in the following. This should be equivalent to the Rayleigh hypothesis, see e.g. van den Berg and Fokkema [10]. For larger corrugations the fields inside the corrugations can be obtained by returning to the integral representations and perform a direct numerical integration. This is quite complicated, however, and will not be pursued. Instead the region inside the corrugations is left blank when the corrugations are not small.

Figure 2 shows the field in the plate for a flat interface and the two orientations of the anisotropy $\psi=45^{\circ}$ and $60^{\circ}$. The direct field from the probe at the angle $45^{\circ}$ is an $\mathrm{S}$ wave. 


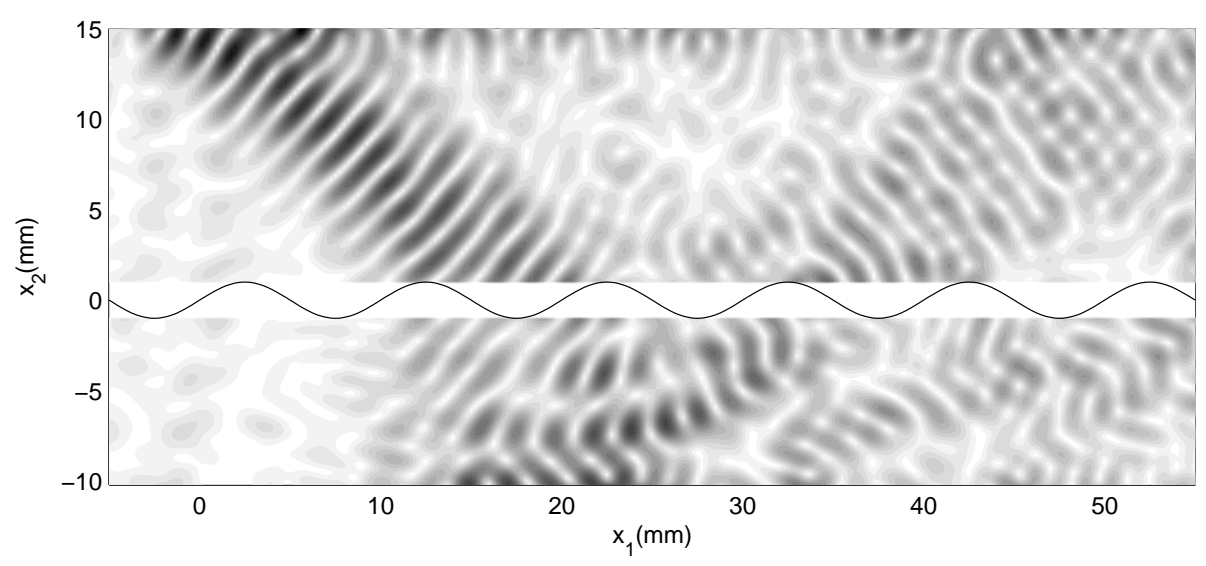

(a) $b=1 \mathrm{~mm}$

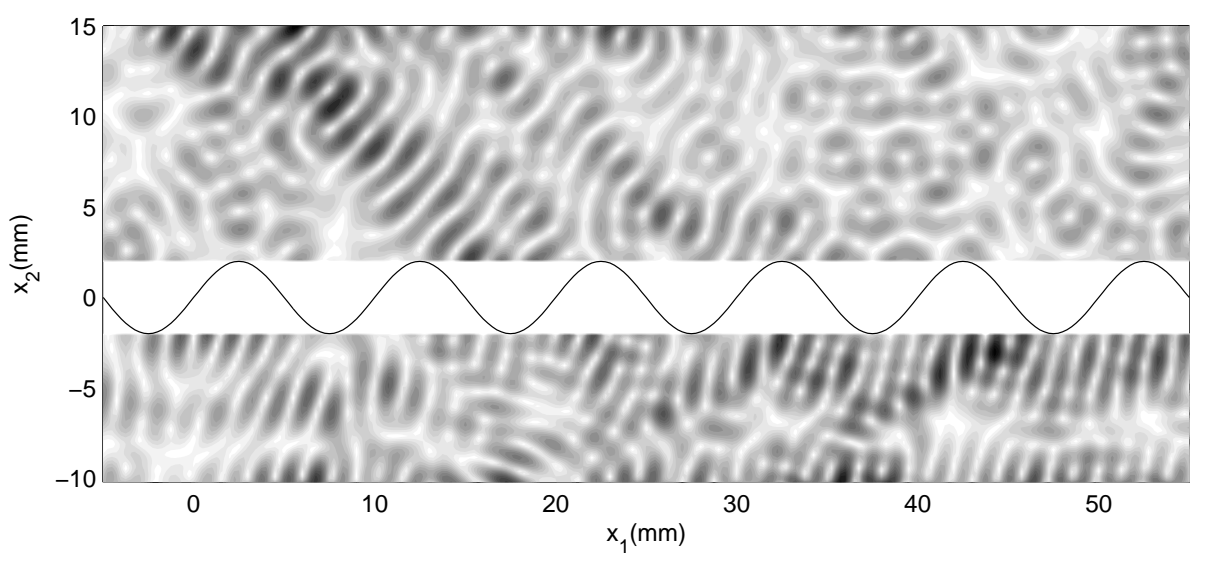

(b) $b=2 \mathrm{~mm}$

FiguRE 6 . The field in the plate with corrugated interface with $a=10 \mathrm{~mm}$ and two different heights $b$. Anisotropy orientation $\psi=60^{\circ}$.

The Rayleigh wave along the surface is also clearly seen. In the lower anisotropic part the wave is more or less propagating vertically, giving a standing wave due to the total reflection from the lower plate surface. The reflected field in the upper part is of course at $45^{\circ}$, but it is shifted somewhat to the right. According to simple ray theory it would hit the upper surface at $x_{1}=42 \mathrm{~mm}$. There is only a relatively small difference in the fields between the two orientations of the anisotropy.

Figure 3 shows the field for $a=5 \mathrm{~mm}$ and $b=0.3$ and $1 \mathrm{~mm}$ for the orientation $\psi=45^{\circ}$. For the smaller corrugation height the field is plotted also inside the corrugations, but for the larger one this is no longer possible (using the expansions in this region very clearly 
leads to erronous, altogether too strong fields). This finding corroborates well with the fact that the Rayleigh hypothesis is well known to be valid for $b / a<0.072$ for the sinusoidal profile, see van den Berg and Fokkema [10]. For $b=0.3 \mathrm{~mm}$ the differences compared to the flat interface are negligible, but for $b=1 \mathrm{~mm}$ there is a clear difference with a reflected field that is actually stronger and more concentrated.

Figure 4 is for the same case as Fig. 3 except that the orientation is $\psi=60^{\circ}$. Also in this case the height $b=0.3 \mathrm{~mm}$ gives negligible differences compared to the flat interface. For $b=1 \mathrm{~mm}$ the differences are larger and the reflected field from the interface is more or less split into parts.

Figure 5 shows the field for $a=10 \mathrm{~mm}$ and $b=1$ and $2 \mathrm{~mm}$ for the orientation $\psi=60^{\circ}$. The Rayleigh hypothesis is not valid so the field is not plotted inside the corrugations. The smaller corrugation height $b=1 \mathrm{~mm}$ has a rather small effect as compared to the flat case, but the higher corrugation $b=2 \mathrm{~mm}$ has a dramatic effect, particularly the field distribution in the lower anisotropic medium is completely altered. It is also noted that there is a directly back-scattered part that interferes with the direct field from the probe.

Figure 6 is for the same case as Fig. 5 except that the orientation is $\psi=60^{\circ}$. Already $b=1 \mathrm{~mm}$ has some effects on the reflected field and for $b=2 \mathrm{~mm}$ the effects are very strong.

\section{CONCLUding REMARKS}

The propagation of elastic waves in a thick plate with two anisotropic materials with a wavy interface is investigated in 2D. The null field approach is employed, which has the advantage compared to integral equations approaches that it is the Fourier transform representation of the Green's tensor that is needed (as opposed to the closed form, which does not exist in the anisotropic case but is preferred in integral equations). Numerical results illuminate the effects of both the anisotropy and the wavy interface. The two materials are reasonably similar (isotropic steel and anisotropic austenitic weld material) and the effects of the wavy interface are then quite small in many cases. But with larger periods and higher amplitudes the effects of the corrugations can be dramatic as is shown in some examples.

The present study is motivated by the need to use ultrasonic nondestructive testing on clad components in the nuclear power industry. But due to processing conditions the cladding is then not $2 \mathrm{D}$ as assumed here. The situation is truly $3 \mathrm{D}$ due to the fact that the crystal axes of the austenite are skewed, so that the assumption that one of the crystal axes is lying perpendicular to the corrugations is no longer valid. It should be straightforward to generalize the present work to this $3 \mathrm{D}$ situation.

\section{ACKNOWLEDGMENT}

The present work is sponsored by the Swedish Nuclear Power Inspectorate (SKI) and this is gratefully acknowledged. 


\section{REFERENCES}

[1] R.J. Hudgell, Handbook on the ultrasonic examination of austenitic clad components, The International Institute of Welding and Joint Research Centre, European Commission, Luxembourg, 1994.

[2] J.T. Fokkema and P.M. van den Berg, Elastodynamic diffraction by a periodic rough surface (stressfree boundary), J. Acoust. Soc. Am. 62 (1977) 1095-1101.

[3] J.T. Fokkema, Reflection and transmission of elastic waves by the spatially periodic interface between two solids (theory of the integral-equation method), Wave Motion 2 (1980) 375-393.

[4] N.E. Glass, R. Loudon, and A.A. Maradudin, Propagation of Rayleigh surface waves across a largeamplitude grating, Phys. Rev. B 54 (1981) 796-805.

[5] A. Boström, Surface waves on the periodic boundary of an elastic half-space, Appl. Sci. Res. 39 (1982) $129-182$.

[6] A. Lakhtakia, V.K. Varadan, V.V. Varadan, and D.N.J. Wall, The T-matrix approach for scattering by a traction-free periodic rough-surface, J. Acoust. Soc. Am. 76 (1984) 1839-1846.

[7] R. Roberts, J.D. Achenbach, R. Ko, L. Adler, A. Jungman, and G. Quentin, Reflection of a beam of elastic waves by a periodic surface profile, Wave Motion 7 (1985) 67-77.

[8] V.K. Varadan, A. Lakhtakia, V.V. Varadan, and C.A. Langston, Radiation characteristics of elastodynamic line source buried in layered media with periodic interfaces. 2. P-wave and SV-wave analysis, Bull. Seismol. Soc. Am. 77 (1987) 2192-2211.

[9] A. El-Bahrawy, Stopbands and passbands for symmetric Rayleigh-Lamb modes in a plate with corrugated surfaces, J. Sound Vib. 170 (1984) 145-160.

[10] P.M. van den Berg and J.T. Fokkema, The Rayleigh hypothesis in the theory of reflection by a grating, J. Opt. Soc. Am. 69 (1979) 27-31.

Department of Applied Mechanics, Chalmers University of Technology, SE-412 96 Göteborg, SwEden 